\title{
Escala de Expectativas de Carreira na Organização: Desenvolvimento e Evidências de Validade
}

\author{
Vinicius Carvalho de Vasconcellos - Universidade de Brasilia, Brasilia, Brasil \\ Elaine Rabelo Neiva - Universidade de Brasilia, Brasilia, Brasil
}

\begin{abstract}
Resumo
Expectativas de carreira figuram como elemento importante para compreender a relação dos profissionais com seu trabalho e organização. Assim, o objetivo deste artigo foi descrever o desenvolvimento e fornecer evidências de validade da Escala de Expectativas de Carreira na Organização, instrumento que afere expectativas dos indivíduos sobre o futuro de suas carreiras na atual organização. O Estudo 1 relata a análise fatorial exploratória do instrumento $(n=183)$ e o Estudo 2 trata da análise fatorial confirmatória conduzida em amostra independente $(n=352)$. Trabalhadores adultos de diversas organizações constituíram as amostras. Os resultados indicaram a presença de dois fatores: Conquistas Profissionais (nove itens; $\rho=0,95 ; \alpha=0,95$ ) e Relação Carreira-Vida Pessoal (quatro itens; $\rho=0,90 ; \alpha=0,90$ ). Concluiu-se que o instrumento apresenta evidências de validade, facilitando análises acadêmicas sobre expectativas de carreira e despontando como ferramenta de diagnóstico útil na gestão de recursos humanos.
\end{abstract}

Palavras-chave: carreira, expectativas, análise fatorial, medidas

\section{Career Expectations in the Organization Scale: Development and Validity Evidence}

\begin{abstract}
Career expectations are an important element to understand the relationship between professionals and their work/organization. The objective of this paper is to describe the procedures for development and provide validity evidence of the Career Expectations in the Organization Scale, an instrument that assesses individuals' expectations about the future of their careers in their respective organizations. Study 1 reports the exploratory factor analysis of the instrument $(n=183)$ and Study 2 describes a confirmatory factor analysis conducted on an independent sample $(n=352)$. Both samples were formed by adult employees of different organizations. The results indicated the presence of two factors: Professional Achievements (nine items; $\rho=.95$; $\alpha=.95$ ) and Career-Personal Life Relationship (four items; $\rho=.90 ; \alpha=.90$ ). The discussion highlights that the scale shows validity evidence, facilitates academic analyzes of the career expectations and is useful as a diagnostic tool in human resources management.

Keywords: career, expectations, factor analysis, measures
\end{abstract}

Escala de Expectativas de Carrera en la Organización: Desarrollo y Evidencias de Validez

\begin{abstract}
Resumen
Expectativas de carrera figuran como un elemento importante para comprender la relación de los profesionales con su trabajo y la organización. El objetivo de este artículo fue describir el desarrollo y proporcionar evidencias de validez de la Escala de Expectativas de Carrera en la Organización, instrumento que mide expectativas de los individuos sobre el futuro de sus carreras en la actual organización. El estudio 1 relata el análisis factorial exploratorio del instrumento $(n=183)$ y el estudio 2 se ocupa del análisis factorial confirmatorio realizado en una muestra independiente $(n=352)$. Participaron trabajadores adultos de diversas organizaciones, y los resultados indicaron la presencia de dos factores: Conquistas Profesionales (nueve ítems; $\rho=0,95 ; \alpha=$ $0,95)$ y Relación Carrera-Vida Personal (cuatro ítems; $\rho=0,90 ; \alpha=0,90$ ). Los resultados indicaron que el instrumento presenta evidencias de validez, facilitando análisis académicos sobre las expectativas de carrera e indicado como herramienta de diagnóstico útil en la gestión de recursos humanos.

Palabras clave: carrera, expectativas, análisis factorial, medidas
\end{abstract}

O exercício de projetar o futuro faz parte da relação dos indivíduos com o trabalho. Expectativas, aspirações e desejos sobre o futuro na carreira comumente emergem no cotidiano laboral e há evidências de que perspectivas de desenvolvimento e crescimento na carreira despontam entre as características mais valorizadas por trabalhadores em organizações (Tolfo, 2002).

O futuro na carreira, embora seja objeto de estudo tradicional da psicologia vocacional e do desenvolvimento de carreira em estudantes (Ourique \& Teixeira,
2012, por exemplo), ainda carece de pesquisas mais sistemáticas no domínio organizacional (Vasconcellos, 2015). Apesar de incipientes, as investigações neste último domínio sugerem o elo entre expectativas e percepções de futuro na carreira e variáveis relevantes para a gestão, como comprometimento organizacional (Chay \& Aryee, 1999; Prince, 2003; Stroh \& Reilly, 1997), satisfação com o trabalho (Burke \& MacDermind, 1999; Chen, Ployhart, Cooper-Thomas, Anderson, \& Bliese, 2011), desligamentos e intenções de desligamento 
(Buckley, Fedor, Veres, Wiese, \& Carraher, 1998; Chay \& Aryee, 1999; Stroh \& Reilly, 1997) e desempenho no trabalho (Zacher \& Frese, 2011),

O contexto contemporâneo coloca o futuro na carreira no centro das atenções dos indivíduos. Décadas atrás, era comum profissionais permanecerem longo tempo na mesma organização, realizando as mesmas atividades e conhecendo de antemão as etapas de sua carreira. Atualmente, contudo, cresce o número de trajetórias profissionais dotadas de grande imprevisibilidade, mobilidade, flexibilidade e autogerenciamento (Arthur, 1994; Gluber, Arnold, \& Coombs, 2014; Hall, 2004). Como o percurso é cada vez menos linear e pré-estabelecido, os indivíduos tendem a monitorar oportunidades profissionais mais atentamente e a refletir constantemente sobre o futuro na carreira, que surge então como questão em aberto.

O cenário contemporâneo e as evidências da literatura mencionadas assinalam a relevância de se estudar, no âmbito organizacional, a visão dos profissionais sobre o futuro de sua carreira. Essa investigação é favorecida caso existam medidas válidas e confiáveis. Nessa linha, o objetivo deste artigo é descrever o desenvolvimento e estudos sobre a validade da Escala de Expectativas de Carreira na Organização (EECO), instrumento que afere expectativas dos indivíduos sobre o futuro de suas carreiras em suas respectivas organizações.

$\mathrm{O}$ artigo revisa, inicialmente, as abordagens e os instrumentos da literatura sobre a temática. A partir da literatura, propõe-se um novo construto e justifica-se o desenvolvimento do novo instrumento. São descritos então dois estudos: o primeiro relata a análise fatorial exploratória da escala e o segundo trata da análise fatorial confirmatória conduzida em amostra independente. $\mathrm{Na}$ última parte do artigo, discute-se o formato final da escala e as contribuições/limitações dessa pesquisa.

\section{Futuro na Carreira em Organizações: Abordagens e Instrumentos}

No âmbito organizacional, o futuro na carreira é estudado pela literatura principalmente por meio de afetos (ansiedade e medo sobre a evolução da carreira, por exemplo), aspirações (futuro desejado, preferido ou planejado) ou expectativas e percepções sobre como provavelmente será o porvir (Vasconcellos, 2015). As pesquisas diferenciam-se também quanto ao ambiente de referência dado aos participantes: pode-se indagar sobre o futuro na atual organização, em outras organizações ou de modo inespecífico (Vasconcellos, 2015). Considerando a natureza da escala proposta neste artigo, a revisão a seguir centra-se em variáveis/instrumentos que aferem expectativas e percepções dos profissionais sobre o futuro de suas carreiras na organização atual.

Mesmo nesse recorte, há distintas formas de investigar e aferir o fenômeno. A mais simples trata de pesquisar o futuro profissional genericamente, isto é, sem explicitar atributos específicos. Tais estudos aferiram expectativas com itens como "Ficarei satisfeito com esse trabalho" (Buckley et al., 1998) ou "Espero que trabalhar nessa organização seja ótimo" (Carr, Pearson, Vest \& Boyar, 2006).

$\mathrm{Na}$ mesma direção, o construto perspectiva de tempo futuro organizacional (Treadway, Breland, Adams, Duke, \& Willians, 2010) não alude a atributos particulares ao abordar o futuro na carreira. Definido como as percepções dos profissionais sobre o tempo que lhes resta em seu papel organizacional, tal construto é aferido por uma escala de 10 itens $(\alpha=0,88)$. "Nesta organização, muitas oportunidades esperam por mim no futuro" constitui um exemplo de item dessa escala.

Nessa última pesquisa, o termo oportunidade(s) é utilizado em itens orientados para o futuro. Contudo, há instrumentos que o utilizam sem aludir nitidamente ao futuro. Assim, Kraimer, Seibert, Wayne, Liden e Bravo (2011) aferiram oportunidades de carreira percebidas com itens como "Existem oportunidades nesta organização atraentes para mim”. Como texto está redigido no tempo presente e não menciona a palavra futuro, os respondentes podem considerar a existência de oportunidades apenas na atualidade. De fato, a percepção sobre oportunidades no presente e as expectativas de oportunidades no futuro não coincidem necessariamente. Por exemplo, profissionais com poucas oportunidades no presente podem vislumbrar oportunidades futuras ao presumir crescimento da organização no porvir.

Para além dessas opções mais genéricas, outros autores analisaram o futuro na carreira a partir de atributos específicos. Neste ponto, destaca-se a mobilidade, isto é, as mudanças de atividade, cargo, ocupação ou organização (Feldman \& Ng, 2007). A variável prospectos de carreira (Burke \& Macdermid, 1999) ilustra tal abordagem ao versar sobre expectativas de promoções em um instrumento de três itens $(\alpha=0,66)$. Por seu turno, Prince (2003) desenvolveu escala de cinco itens $(\alpha=0,82)$ que avalia expectativas de mobilidade vertical/promoções ("Nos próximos dois anos, terei a chance de assumir uma posição mais alta no meu cargo"), mas também de mobilidade horizontal, ou seja, mudança de cargo no mesmo nível hierárquico ("Nos 
próximos dois anos, terei a chance de mudar para um cargo similar em outra localidade ou área").

Nesse mesmo estudo, Prince (2003) introduziu uma medida sobre expectativas de melhoria de papel, isto é, projeções sobre progressos nas atividades realizadas. A escala $(\alpha=0,82)$ contém sete itens como, por exemplo, "Nos próximos dois anos, minhas tarefas exigirão que eu aja de forma mais independente". Inversamente, outros autores interessaram-se pela percepção de que o conteúdo das atividades seria limitado no futuro (Eby, Allen, \& Brinley, 2005). Entre os seis itens dessa escala $(\alpha=0,78)$ estão: "As tarefas do meu trabalho atual se tornarão rotina no futuro" e "Minhas responsabilidades no trabalho crescerão significativamente no futuro" (pontuação reversa).

Outro atributo trazido à cena são as expectativas salariais. Gibson e Lawrence (2010), por exemplo, operacionalizaram a variável expectativa de carreira por meio do nível salarial esperado pelos profissionais ao se aposentar/desligar da organização. De modo similar, O’Neill, Stanley e O’Reilly (2011) mediram o quanto indivíduos no início da trajetória profissional esperavam receber de salário no topo de suas carreiras. Diferente de Gibson e Lawrence (2010), a medida era inespecífica quanto ao ambiente organizacional de desenvolvimento da carreira. Nessas pesquisas, os autores não construíram escalas, optando por medidas diretas e de item único na coleta de dados.

Já a variável expectativas de trabalho (Chen et al., 2011), definida como expectativas dos profissionais sobre as experiências e condições futuras do trabalho atual, conjuga diversos atributos. A escala de 12 itens $(\alpha=0,90)$ abrange projeções de salário, de mobilidade/ desenvolvimento de carreira e de desafios no trabalho (atributos presentes em pesquisas já citadas), além de expectativas sobre relações com pares/chefia e condições de trabalho. Os respondentes estimavam como esses aspectos do trabalho mudariam nos meses seguintes (variando entre piorarão muito até melhorarão muito).

$\mathrm{Na}$ esfera nacional, o futuro na carreira é examinado notadamente por meio de entrevistas e pesquisas qualitativas (Diogo, 2007; Graf \& Coutinho, 2010; Lopes \& Silva, 2009). Excluindo o estudo de Lopes e Silva (2009), os demais se ocuparam de aspirações (e não de expectativas/percepções) e apenas tangenciaram o futuro na carreira, posto que foram centradas em outras agendas de investigação.

Em abordagens quantitativas, nota-se o predomínio de estudos sobre percepções de oportunidades de crescimento na carreira no tempo presente (por exemplo, Brandão \& Bastos, 1993). Pesquisas recentes (Veloso et al., 2011; Veloso, Silva \& Dutra, 2012) aferiram percepções de crescimento na carreira na organização com os seguintes itens: "Acredito que trabalhando nesta empresa terei oportunidade de fazer carreira e crescer", "Sei o que devo fazer para crescer profissionalmente nesta empresa" e "Considero justos os critérios de promoção e carreira adotados nesta empresa". Entre esses itens apenas o primeiro aborda, de fato, expectativas de futuro na carreira.

Entre as escalas nacionais orientadas para o futuro, cita-se a Escala de Expectativa de Futuro (Souza, Pereira, Funck, \& Formiga, 2013). Contudo, esta escala não está centrada na vida profissional, pois captura também expectativas sobre o desenvolvimento da sociedade e de aspectos da vida pessoal.

\section{Proposição da Escala de Expectativas de Carreira na Organização}

Após revisar a literatura, optou-se aqui por formular uma proposta integradora, mas que fosse, concomitantemente, capaz de delimitar fronteiras nítidas para o objeto de pesquisa. Assim, conforme exposto na introdução, este artigo propõe-se um novo construto, denominado de expectativas de carreira na organižação. O construto é definido como as crenças dos indivíduos sobre sua futura trajetória profissional na atual organização.

Em um cenário de carreira sem fronteiras (Arthur, 1994), a referência à atual organização pode gerar estranhamento. Contudo, há indícios que essa opção predomina nos estudos sobre futuro na carreira (Vasconcellos, 2015). Provavelmente, tal padrão relaciona-se com a suposição que esse enfoque fornece um horizonte mais concreto para se projetar o futuro (sendo assim valorizado pelos pesquisadores), além de propiciar insumos mais diretos para as organizações (sendo mais atraente para os gestores e possibilitando mais estudos). Em adição, estudar expectativas de carreira na organização não se contrapõe ao modelo de carreira sem fronteiras. Ao contrário, pode ajudar a compreendê-lo, dado que expectativas desfavoráveis na atual organização podem incitar os indivíduos a desenvolver suas carreiras neste modelo.

O termo expectativas está entre os mais empregados nos estudos sobre futuro na carreira (Chen et al., 2011; Gibson \& Lawrence, 2010; O’Neill et al., 2011; Prince, 2003) e sua utilização no construto reforça a orientação para o futuro. Expectativas refletem crenças sobre a probabilidade de ocorrência de um situação/ 
evento no futuro (Oettingen \& Mayer, 2002). Assim, expectativas de carreira referem-se às crenças sobre a probabilidade de determinadas situações ou estados ocorrerem no futuro profissional. Essas situações ou estados constituem, na prática, os atributos da EECO. A aferição de expectativas traz ainda vantagens sobre a mensuração de oportunidades e possibilidades pois evita a imprecisão quanto à orientação temporal desses últimos termos. Em adição, a aferição de expectativas captura o caso de profissionais com carreiras consolidadas que detêm visão positiva do futuro pela simples manutenção de sua posição, sem necessidade de novas oportunidades e possibilidades.

A revisão de literatura da seção anterior possibilitou ainda sumariar os principais instrumentos e atributos utilizados ao se abordar o futuro na carreira. Para além das avaliações generalistas (Buckley et al., 1998; Carr et al., 2006) e das projeções sobre o grau de abertura (existência de possibilidades/oportunidades) com o qual o futuro se apresenta (Treadway et al., 2010; Zacher \& Frese, 2011), os autores pautaram-se em mobilidade de cargos/funções (Burke \& Macdermid, 1999; Prince, 2003; Veloso et al., 2011), remuneração/ salários (Chen et al., 2011; Gibson \& Lawrence, 2010; O’Neill et al., 2011), características das atividades (Chen et al., 2011; Eby et al., 2005; Prince, 2003) e relações/ condições de trabalho (Chen et al., 2011).

Ao se analisar tais estudos, nenhum instrumento parece delimitar, de forma completa e precisa, as expectativas de carreira na organização. Pesquisas alicerçadas em avaliações generalistas e no grau de abertura do futuro na carreira são demasiadamente inespecíficas e impedem o discernimento de eventuais diferenças nos atributos, fornecendo poucos insumos para a gestão. Estudos que valorizam apenas um atributo (mobilidade, remuneração ou características das atividades) expressam visões parciais do fenômeno. A proposta mais completa (Chen et al., 2011), por sua vez, inclui expectativas sobre relações/condições de trabalho, aspectos que concernem mais ao futuro da organização (ou da equipe de trabalho) do que à carreira do indivíduo.

No Brasil, a carreira tende a ser analisada em questionários sobre clima organizacional e os poucos itens sobre carreira estão geralmente orientados para o presente e para práticas/políticas de recursos humanos, com acento nas promoções (Veloso et al., 2011; Veloso, Silva, \& Dutra, 2012). Recentemente, ocorreu a publicação de uma escala sobre percepção de sucesso na carreira (Costa, 2014), contudo, esta não trata do futuro e não compartilha dos mesmos atributos aqui endossados. De sua parte, a Escala de Expectativa de Futuro (Souza et al., 2013), embora voltada para o futuro, afere expectativas em geral (não apenas sobre trabalho e carreira) e está dirigida prioritariamente para estudantes (e não para profissionais atuando em organizações).

Por sua vez, a EECO parte de um conjunto de atributos que permite visão mais completa do fenômeno, ao contrário das escalas internacionais que tendem a mensurar atributos isolados. Ademais, a EECO, ao aferir expectativas de carreira na organização, surge como instrumento delineado para o mundo organizacional e orientado para o futuro, em contraposição à inclinação geral das escalas nacionais, centradas no momento atual da carreira. Considerando as limitações da literatura, a EECO coloca-se como alternativa diante das escalas anteriores. Desta feita, as próximas seções descrevem os dois estudos que compuseram o desenvolvimento da escala.

\section{Estudo 1}

O objetivo do Estudo 1 foi construir os itens da escala e submetê-los à análise fatorial exploratória. Para redação dos itens, recorreu-se primeiramente à revisão de literatura descrita anteriormente e a uma pesquisa exploratória empreendida no país (Vasconcellos \& Neiva, 2014) sobre os principais aspectos considerados pelos indivíduos no seu futuro profissional na atual organização. Os resultados apontaram projeções de crescimento na carreira (isto é, lograr sucesso, avanços e conquistas profissionais, notadamente por meio de mobilidade), de melhoria na remuneração/recompensas e de obtenção de experiências futuras positivas no trabalho (conseguir realização/reconhecimento profissional e tarefas com significado pessoal) como os principais atributos a ancorar uma visão favorável da carreira no futuro.

$\mathrm{Na}$ escala, as expectativas de crescimento na carreira foram traduzidas por itens sobre o alcance de progressos/conquistas profissionais e o cumprimento de objetivos na carreira. Essa opção segue outros estudos da literatura (Prince, 2003) e respeita a ampla gama de possibilidades de desenvolvimento de carreira, visto que os respondentes podem optar por não priorizar promoções no percurso profissional. Outros aspectos abordados na pesquisa exploratória e na literatura, como projeções de remuneração e recompensas e de experiências positivas no trabalho, também foram contemplados na escala. Neste último caso, foram incluídos atributos como reconhecimento, prestígio e a 
realização de tarefas dentro dos interesses profissionais dos indivíduos.

A inclusão de atributos à margem da ideia tradicional de crescimento na carreira (promoções e remuneração) relaciona-se à emergência, nos últimos decênios, de novas formas de trajetórias profissionais (cada vez menos lineares e verticais, dificultando promoções) e de novos direcionadores na condução das carreiras (como realização profissional e relação carreira-vida pessoal). Artigos teóricos associam tais atributos à ideia de sucesso subjetivo/psicológico (alcance de objetivos com sentido pessoal), aspecto que ganha crescente valorização no contexto contemporâneo (Hall \& Chandler, 2004) e que justifica sua incorporação na EECO.

A relação carreira-vida pessoal, na verdade, não foi observada nos construtos/instrumentos revisados e, embora presente na pesquisa exploratória citada (Vasconcellos \& Neiva, 2014), não constou entre os principais atributos. Todavia, sua inclusão justifica-se teoricamente. Nesta linha, o modelo arco-íris da vida e carreira (Super, 1980) aponta que o desenvolvimento da carreira ocorre por meio da relação entre os diversos papéis sociais desempenhados ao longo da vida (trabalhador, cônjuge, cidadão, entre outros), sinalizando que a trajetória profissional está entremeada pela vida pessoal. Em reforço, recente revisão no campo da carreira argumenta que atentar para interdependência do trabalho e da vida familiar/doméstica é fundamental para compreender as atuais trajetórias profissionais (Greenhaus \& Kossek, 2014).

\section{Método}

\section{Participantes}

A amostra foi composta por 183 profissionais de diversas organizações. Superou-se assim o mínimo de 100 casos e a relação de 10 casos por item (foram 16 itens no total, conforme explicitado a seguir) requerido para esse tipo de análise (Hair, Black, Babin, Anderson \& Tatham, 2010; Pasquali, 2010). Todos os participantes apresentavam escolaridade igual ou maior do que nível médio, a idade média foi de 38,39 anos $(\mathrm{DP}=11,21), 63 \%$ eram mulheres e $72 \%$ não exerciam cargo gerencial.

\section{Instrumento}

A partir da definição do construto e da revisão da literatura, foram escritos 22 itens que convidavam o participante a pensar sobre seu futuro profissional na atual organização. Os itens aludiam a um estado futuro positivado (exemplo: "Serei um profissional bem-sucedido na minha carreira") e o participante assinalava na escala o quão provável seria tal estado/situação, supondo sua permanência naquela organização pelos próximos cinco anos. A escala continha sete pontos e era rotulada nas extremidades $(1$ - nada provável até 7 - extremamente prováveb). Esse formato contempla na escala o caráter probabilístico das expectativas e provê referência temporal aos participantes. A indicação de um ponto específico no futuro facilita a emissão das respostas, pois oferece horizonte concreto para as expectativas dos profissionais. O período de cinco anos justifica-se porque não força o respondente a pensar em prazos muito longos (evitando inconsistências), além de já ter sido utilizado na literatura de futuro na carreira (De Souza, 2002).

O conjunto inicial de itens foi submetido à análise de juízes e à análise semântica. $\mathrm{Na}$ primeira, seis especialistas do campo (professores, mestres e doutorandos em psicologia organizacional ou administração) avaliaram os itens quanto a sua adequação diante do construto e seu grau de clareza. Foram retidos os itens que apresentaram $80 \%$ ou mais de avaliações positivas dos juízes. A análise semântica consistiu em pré-testes dos itens com vistas a verificar sua inteligibilidade em uma pequena amostra da população-alvo (26 profissionais de diferentes organizações e com pelo menos nível médio completo). Como a escala demanda certo grau de abstração, recomenda-se sua aplicação em profissionais com pelo menos esse nível de escolaridade. Após a análise de juízes e semântica, seis itens foram suprimidos, pois foram considerados vagos, repetitivos ou dispensáveis. Assim, além das variáveis demográficas, o questionário contou com 16 itens sobre expectativas de carreira para a análise fatorial exploratória.

\section{Procedimentos}

A pesquisa foi divulgada por meio de listas de e-mails e redes sociais. $\mathrm{Na}$ divulgação, constava um link de acesso que direcionava os participantes para a página eletrônica com o questionário, que foi respondido individualmente. Na página inicial, ao solicitar aos respondentes o consentimento para participar do estudo, foram informados os objetivos, o tempo de duração e o caráter voluntário da pesquisa, assim como os contatos e a filiação institucional dos pesquisadores. Firmou-se também o compromisso de que as respostas permaneceriam no anonimato. 


\section{Análise de dados}

Após tratamento de casos extremos/atípicos e dados faltantes, procedeu-se à inspeção das matrizes de correlações e anti-imagem e à aplicação dos testes de Barlett e de Kaiser-Meyer-Olkin (KMO). A definição do número de fatores foi realizada por análise de componentes principais e considerou múltiplos critérios: a análise de scree plot, o critério de Kaiser-Guttman (retenção de fatores com autovalor $>1$ ), o percentual de variância explicada e a análise paralela (comparação de autovalores empíricos e randômicos). Os autovalores randômicos foram calculados pelo programa Monte Carlo PCA. A análise paralela tende a exibir vantagem diante de critérios tradicionais por fornecer indicação mais precisa sobre o número de fatores a reter (Damásio, 2012). Após estipular a quantidade de fatores, optou-se pelo método de extração Principal Axis Factoring (rotação Promax). A análise fatorial exploratória foi realizada no SPSS 19.

\section{Resultados e Discussão}

Considerando o conjunto de 16 itens, constatou-se que o determinante da matriz registrou um valor próximo, contudo, diferente de zero. Todas as correlações item-item foram significativas $(p<0,05)$ e $88 \%$ superaram 0,30. As correlações anti-imagem foram baixas (93\% abaixo de 0,30, em módulo), configuração favorável para realização de análise fatorial (Tabachnick \& Fidell, 2007). O KMO foi de 0,90, avaliado como meritório (Hair et al., 2010) e o teste de Bartlett foi significativo $\left(\chi^{2}=2249,93 ; p<0,01\right)$.

As avaliações do scree plot, do critério de Kaiser-Guttman e do percentual de variância explicada indicaram solução com até três fatores. Os autovalores e o percentual de variância explicada dos três fatores foram respectivamente: 8,43 (52,65\%), 2,29 (14,29\%) e 1,25 (7,79\%). A análise paralela apontou a existência de dois fatores, pois o autovalor empírico do terceiro fator $(1,25)$ foi inferior ao autovalor aleatório correspondente $(1,34)$. Considerando o resultado da análise paralela, o alto percentual de variância explicada com apenas dois fatores $(66,93 \%)$ e a busca por parcimônia, foi escolhida a solução bidimensional.

$\mathrm{Na}$ análise fatorial exploratória (Principal Axis Factoring), dois itens obtiveram comunalidades inferiores a 0,50 e foram excluídos visto que prejudicariam uma estrutura fatorial bem definida (Hair et al., 2010). A nova matriz (14 itens) permaneceu fatorável e o caráter bidimensional tornou-se mais claro (além da análise paralela, o scree plot e o critério de Kaiser-Guttman passaram também a indicar dois fatores). A Tabela 1 expõe as cargas fatoriais da solução de dois fatores. Todas superaram 0,50 e apenas duas registram valores inferiores a 0,70 . Todas as comunalidades excederam 0,50. A solução com dois fatores explicou, no total, $65 \%$ de variância dos dados.

$\mathrm{O}$ primeiro fator (F1 na Tabela 1) foi denominado de Conquistas Profissionais. Os itens com maior carga nesse fator tratam da consecução de conquistas/ objetivos de carreira e parecem funcionar como eixo para o qual convergem elementos tipicamente considerados pelos indivíduos em seu futuro profissional: recompensa/remuneração, prestígio/reconhecimento e obtenção de tarefas interessantes e alinhadas às preferências pessoais. Tais elementos estão entre os mais citados na pesquisa exploratória mencionada e expressam atributos contemplados na literatura (Chen et al., 2011; Gibson \& Lawrence, 2010; O’Neill et al., 2011; Prince, 2003; Vasconcellos \& Neiva, 2014).

O segundo fator (F2 na Tabela 1) foi nomeado de Relação Carreira-Vida Pessoal. Abarca itens relativos às expectativas de obtenção de uma relação favorável entre a vida privada e o trabalho/carreira no futuro. Sob essa ótica, faz emergir o conjunto de efeitos recíprocos entre essas duas esferas, distinguindo-se, portanto, do primeiro fator. Assim, contempla-se no instrumento a relação entre os papéis associados ao trabalho e à vida pessoal, tema salientado por contribuições teóricas da literatura sobre carreira (Greenhaus \& Kossek, 2014; Super, 1980).

O alfa de Cronbach dos fatores suplantou o patamar mínimo de 0,70 (Damásio, 2012; Field, 2009; Hair et al., 2010). Outras medidas de fidedignidade foram calculadas, a saber, as médias das correlações interitem e das correlações item-total corrigidas (Field, 2009; Hair et al., 2010). Hair et al. (2010) recomendam 0,30 e 0,50 como patamares mínimos para as médias das correlações interitem e item-total corrigidas, respectivamente. Os fatores extraídos ultrapassaram essas referências (Tabela 1).

\section{Estudo 2}

O objetivo do Estudo 2 foi submeter a EECO à análise fatorial confirmatória, a fim de se testar a estrutura revelada no Estudo 1.

\section{Método}

\section{Participantes}

Na nova amostra coletada $(n=352)$, a idade média foi de 42,26 anos (DP $=12,57), 51 \%$ eram homens e $58 \%$ não exerciam cargo gerencial. Os respondentes 
Tabela 1

Cargas Fatoriais na Análise Fatorial Exploratória da EECO

\begin{tabular}{lcc}
\hline Item & F1 & F2 \\
\hline 5. Terei conseguido conquistas profissionais relevantes na minha carreira. & 0,91 \\
15. Atingirei meus objetivos de carreira estabelecidos para esse período. & 0,82 \\
3. Serei bem remunerado em relação às minhas responsabilidades profissionais. & 0,79 \\
9. Estarei satisfeito com o progresso da minha carreira. & 0,79 \\
16. Serei um profissional bem-sucedido na minha carreira. & 0,77 \\
1. Serei reconhecido pela organização por minhas contribuições no trabalho. & 0,77 \\
6. Minha remuneração será compatível com minhas expectativas de salário para esse período. & 0,74 \\
2. Terei prestígio entre meus colegas em função do trabalho realizado. & 0,70 \\
7. Minhas atividades profissionais serão mais interessantes do que hoje. & 0,69 \\
10. Realizarei um trabalho compatível com minhas preferências profissionais. & 0,57 \\
17. Minha vida pessoal estará preservada de interferências do trabalho. & 0,94 \\
19. Minha carga horária de trabalho será compatível com minhas necessidades pessoais. & 0,91 \\
18. Terei boa qualidade de vida mesmo considerando as exigências do trabalho. & 0,84 \\
12. Conseguirei equilíbrio entre vida pessoal e trabalho. & 0,83 \\
Percentual de variância explicada (\%) & 13,85 \\
Confiabilidade (alfa de Cronbach) & 50,94 \\
Média das correlações interitem & 0,94 \\
Média das correlações item-total corrigidas & 0,93 \\
\hline
\end{tabular}

Nota. EECO = Escala de Expectativas de Carreira na Organização. São exibidas apenas as cargas fatoriais acima de 0,30.

apresentavam escolaridade igual ou superior ao nível médio e trabalhavam em diversas organizações (cerca de metade da amostra atuava em empresas privadas). O recrutamento dos participantes foi realizado por consultoria especializada contratada para esse fim.

\section{Instrumento}

Os participantes responderam aos 14 itens da EECO dispostos na solução fatorial final do Estudo 1. Ademais, o questionário contava com itens demográficos.

\section{Procedimentos}

A consultoria enviou correio eletrônico para os potenciais respondentes com um link para a página eletrônica da pesquisa (taxa de resposta de $25 \%$ ), na qual constavam as instruções e o questionário. Este foi respondido individualmente, de forma voluntária e anônima. A página inicial do questionário replicou o padrão reportado no Estudo 1. Por meio da consultoria contratada, os participantes receberam cerca de $\mathrm{R} \$$ 15 reais como retribuição pela participação no estudo, prática corriqueira no exterior. Desde que tal incentivo não seja excessivo (como no caso da presente pesquisa), tal prática está condizente com os princípios éticos que regem a pesquisa internacional em psicologia (American Psychological Association, 2010).

\section{Análise de dados}

Após tratamento de casos extremos/atípicos e dados faltantes, executou-se a análise fatorial confirmatória (estimação por máxima verossimilhança). Como os dados não exibiram normalidade multivariada (com base no índice de Mardia), recorreu-se à técnica de bootstrapping (500 subamostras foram geradas) para se avaliar a estabilidade dos parâmetros estimados (Byrne, 2010).

Para determinar a estrutura fatorial que melhor se ajustava à EECO, os seguintes indicadores foram observados: GFI (Goodness-of-Fit Index), CFI (Comparative Fit Index), RMSEA (Root Mean Square Error of Approximation), SRMR (Standardized Root Mean Residual) e a diferença entre os valores de $\chi^{2}$ (qui-quadrado) entre modelos. Além do alfa de Cronbach, a confiabilidade do construto foi calculada também pelo $\operatorname{rho}(\rho)$ de 
Jöreskog. Utilizou-se o programa AMOS 18 na análise fatorial confirmatória.

\section{Resultados e Discussão}

Os resultados iniciais da análise fatorial confirmatória indicaram que o item 1 ("Serei reconhecido pela organização por minhas contribuições no trabalho") exibiu resíduos padronizados elevados com alguns itens, sendo então suprimido. Além desse critério estatístico, a supressão do item 1 apoiou-se nos seguintes pontos: (a) seu conteúdo está coberto em boa medida pelo item 2, (b) seu conteúdo não apareceu entre os principais atributos revelados pela revisão de literatura e pela pesquisa exploratória mencionada e (c) o fator Conquistas Profissionais já continha boa quantidade de itens.

Após a exclusão do item, três modelos foram gerados. Inicialmente, testou-se um modelo de fator único, prática recomendada quando não há indicação teórica sobre o número de fatores (Kline, 2011). Examinou-se então o modelo de dois fatores do Estudo 1. Por fim, o terceiro modelo reflete versão reduzida desse último modelo. Esta versão foi proposta para diminuir, em pesquisas futuras, o tamanho do questionário a ser respondido pelos participantes quando a
EECO for aplicada junto a outras escalas. Para compor a versão reduzida foram observados os seguintes critérios: (a) os principais atributos expostos pela revisão de literatura e pesquisa exploratória deveriam continuar representados; (b) os itens suprimidos deveriam contar, de preferência, com conteúdo coberto parcialmente por itens remanescentes e pertencer ao fator Conquistas Profissionais (que abriga nove itens) e (c) os itens suprimidos deveriam estar entre aqueles com maiores resíduos padronizados. $\mathrm{O}$ modelo reduzido (detalhado posteriormente) contou com 10 itens distribuídos nos mesmos fatores do modelo completo de dois fatores do Estudo 1.

$\mathrm{O}$ modelo de fator único manifestou ajuste ruim: $\chi^{2}(g l)=596,07(63), p<0,01, \mathrm{GFI}=0,75, \mathrm{CFI}=0,87$, RMSEA $=0,16$ e SRMR $=0,08$. Já o modelo completo de dois fatores apresentou os seguintes indicadores: $\chi^{2}(g l)=209,79(62), p<0,01, \mathrm{GFI}=0,92, \mathrm{CFI}=0,96$, RMSEA $=0,08$ e SRMR $=0,04$. A diferença de qui-quadrado entre esses modelos, $\Delta \chi_{(1)}^{2}=386,28, p<0,01$, foi elevada e significativa, reforçando a existência de dois fatores. De sua parte, o modelo reduzido de dois fatores exibiu os indicadores a seguir: $\chi^{2}(g)=129,49(34), p<$ 0,01, GFI $=0,93$, CFI $=0,96$, RMSEA $=0,09$ e SRMR $=0,04$. Ambos os modelos de dois fatores (completo e reduzido) exibiram ajustes adequados considerando os

Tabela 2

Cargas Fatoriais na Análise Fatorial Confirmatória da EECO

\begin{tabular}{lccc}
\hline Fator & Item & $\begin{array}{c}\text { Modelo de Dois Fatores } \\
\text { (completo) }\end{array}$ & $\begin{array}{c}\text { Modelo de Dois Fatores } \\
\text { (reduzido) }\end{array}$ \\
\hline Conquistas Profissionais & Item 2 & 0,70 & 0,70 \\
& Item 3 & 0,75 & - \\
& Item 5 & 0,85 & 0,87 \\
& Item 6 & 0,75 & 0,76 \\
& Item 7 & 0,84 & 0,83 \\
& Item 9 & 0,93 & - \\
& Item 10 & 0,84 & 0,82 \\
& Item 15 & 0,87 & 0,86 \\
& Item 16 & 0,84 & - \\
Relação Carreira-Vida Pessoal & Item 12 & 0,82 & 0,82 \\
& Item 17 & 0,82 & 0,82 \\
& Item 18 & 0,89 & 0,89 \\
& Item 19 & 0,82 & 0,82 \\
\hline
\end{tabular}

Nota. EECO = Escala de Expectativas de Carreira na Organização. Todas as cargas são estatisticamente significativas $(p<0,01)$. 
parâmetros da literatura (Hair et al., 2010; Tabachnick \& Fidell, 2007).

Com base nesses elementos, a estrutura de dois fatores foi confirmada como a mais adequada. Assim, a Tabela 2 explicita a composição final dos modelos de dois fatores (completo e reduzido) e as cargas fatoriais padronizadas de seus itens. Todas as cargas fatoriais foram estatisticamente significativas nos modelos $(p<0,01)$.

As médias das cargas fatoriais das subamostras do bootstrapping foram praticamente idênticas às cargas fatoriais da Tabela 2 (a diferença máxima não alcançou nem 0,01 ), com baixo erro padrão (entre 0,01 e 0,03 ). Esses resultados, presentes tanto no modelo completo como no reduzido, demonstram a estabilidade das cargas e fornecem evidência sobre a adequação do processo de estimação dos modelos.

Para testar a validade de construto dos modelos completo e reduzido, foram examinadas as validades convergente e discriminante, seguindo as indicações de Hair et al. (2010) e Kline (2011). Na validade convergente, três pontos foram abordados. O primeiro foi a magnitude das cargas fatoriais dos itens em cada fator. Conforme Tabela 2, todas as cargas fatoriais igualam ou ultrapassam o patamar sugerido 0,70 para soluções fatoriais ótimas. O segundo ponto foi a variância média extraída por fator. Os valores dos fatores Conquistas Profissionais (0,67 e 0,66 no modelo completo e reduzido, respectivamente) e Relação Carreira-Vida Pessoal $(0,70$ nos dois modelos) extrapolam 0,50 , referência que indica convergência adequada. $\mathrm{O}$ terceiro ponto foi a confiabilidade do construto, calculada pelas medidas rho de Jöreskog e alfa de Cronbach. A confiabilidade de Conquistas Profissionais no modelo completo $(\rho=$ $0,95 ; \alpha=0,95)$ e no reduzido $(\rho=0,92 ; \alpha=0,92)$, assim como do fator Relação Carreira-Vida Pessoal ( $\rho=0,90$; $\alpha=0,90$ em ambos os modelos), suplantam 0,70, limiar que indica bons níveis de confiabilidade. Esses três pontos indicam a validade convergente da medida.

$\mathrm{Na}$ validade discriminante, mensura-se até que ponto os fatores são diferentes. Nos dois modelos, tal análise torna-se particularmente relevante dada a correlação entre os fatores, que atingiu 0,73 no modelo completo e 0,74 no modelo reduzido $(p<0,01 \mathrm{em}$ ambos os casos). Em modelos bem definidos, a correlação entre fatores deve ser inferior a 0,90 (Kline, 2011). Portanto, a correlação entre fatores nos dois modelos fornece evidência de validade discriminante. Outro indício de validade discriminante emerge quando as variâncias médias extraídas dos fatores excedem o quadrado da correlação entre eles, posto que o construto deve explicar melhor seus itens do que outro construto. As variâncias médias extraídas dos fatores no modelo completo $(0,67$ e 0,70$)$ superam o quadrado de sua correlação $\left(0,73^{2}=0,53\right)$. No modelo reduzido, as variâncias médias extraídas dos fatores $(0,66$ e 0,70$)$ também excedem o quadrado de sua correlação $\left(0,74^{2}=0,55\right)$. Corrobora-se assim, nos dois modelos, a validade discriminante.

\section{Considerações Finais}

Partindo das lacunas da literatura, este artigo propôs o construto expectativas de carreira na organização, descreveu a construção de uma escala para mensurá-lo e apresentou evidências de validade/confiabilidade. Entre suas características, a EECO adota clara orientação para o futuro, afere expectativas de profissionais sobre sua carreira na atual organização e recobre adequadamente as facetas do fenômeno. Distingue-se, portanto, de instrumentos orientados para o presente e para práticas/políticas de recursos humanos (Veloso et al., 2011; Veloso, Silva \& Dutra, 2012) e de escalas que englobam expectativas sobre vários aspectos da vida pessoal e social direcionadas para estudantes (Souza et al., 2013).

A estrutura fatorial encontrada sugere a existência de dois conjuntos de expectativas. O primeiro fator agrupou conquistas no ambiente de trabalho, incluindo atributos como remuneração, prestígio entre pares, avanços/crescimento/sucesso na carreira e realização de atividades interessantes e alinhadas às preferências individuais. Embora alguns desses atributos tenham sido abordados isoladamente em pesquisas anteriores (Burke \& Macdermid, 1999; Gibson \& Lawrence, 2010; O’Neill et al., 2011; Prince, 2003), a confluência neste fator sugere o caráter imbricado dessas expectativas. $\mathrm{O}$ segundo fator versa sobre a relação da carreira/trabalho com a vida pessoal/privada. Seus itens alinham-se a estudos teóricos e empíricos que, ao discutir a carreira contemporânea, frisam a importância de sua interface com a vida fora do trabalho, a obtenção do sucesso psicológico e a realização de tarefas com sentido pessoal (Greenhaus \& Kossek, 2014; Hall \& Chandler, 2004; Prince, 2003). Como as duas versões da escala possuem bons índices e o mesmo número de fatores, recomenda-se, de modo geral, a utilização da versão reduzida por ser mais parcimoniosa.

O presente estudo contribui para a academia e a gestão das organizações ao apresentar escala que porta 
visão abrangente e integrada das expectativas de carreira, com bons índices de validade e confiabilidade, adaptada ao contexto brasileiro e rapidamente aplicada. Em consequência, a EECO pode auxiliar investigações acadêmicas sobre os antecedentes e consequentes das expectativas de carreira e colaborar na compreensão da intrincada ligação indivíduo-trabalho-organização, inclusive no teste de modelos teóricos.

Em adição, a EECO pode ser empregada em organizações para diagnosticar as expectativas dos profissionais sobre seu futuro. Como exposto na introdução, a imprevisibilidade nos vínculos de trabalho, a crescente mobilidade e a maior responsabilidade do indivíduo na gestão da carreira contemporânea incitam os trabalhadores a monitorar oportunidades e a refletir sobre o futuro constantemente. Por essa razão, a análise das expectativas de carreira dos profissionais pode aprimorar a gestão de recursos humanos nas organizações. Com efeito, tais expectativas relacionam-se com atitudes no trabalho e intenções de desligamento (Chay \& Aryee, 1999; Chen et al., 2011; Prince, 2003; Stroh \& Reilly, 1997), dados que reforçam sua relevância.

Os resultados da EECO devem ser apurados respeitando sua estrutura fatorial e calculados individualmente por fator a partir da média aritmética simples de seus itens. $\mathrm{Na}$ interpretação dos dados, deve-se considerar que quanto maior for o escore obtido, maior a probabilidade de ocorrência das situações/estados descritos pelos itens na visão dos respondentes. Como a redação dos itens adquire sempre conotação positiva, escores maiores sinalizam expectativas mais favoráveis. A EECO pode ser aplicada por psicólogos, administradores ou outros profissionais da área de recursos humanos, desde que as orientações presentes neste artigo sejam respeitadas.

Entre as limitações deste estudo, informa-se que a EECO, na atual configuração, deve ser aplicada apenas em profissionais com pelo menos nível médio de escolaridade. Outra limitação concerne à necessidade de novas amostras para validar a solução fatorial descortinada, posto que os resultados aqui expostos são mais indicativos do que conclusivos. O caráter não aleatório das amostras constitui outra limitação.

De todo modo, é provável que a EECO fomente novas investigações sobre expectativas de carreira. Analisar a relação entre trajetória pregressa dos indivíduos, suas atitudes/afetos sobre a atual situação de trabalho e suas expectativas de carreira configura possibilidade promissora de pesquisa. A literatura ainda devota pouca atenção às variáveis e aos construtos relacionados ao tempo. Espera-se que a publicação da EECO contribua, dentro de suas possibilidades, para reverter esse quadro.

\section{Referências}

American Psychological Association. (2010). Ethical principles of psychologists and code of conduct. Recuperado de http://www.apa.org/ethics/code/\#805

Arthur, M. B. (1994). The boundaryless career: A new perspective for organizational inquiry. Journal of Organizational Behavior, 15(4), 295-306. doi: 10.1002/ job.4030150402

Brandão, M. G., \& Bastos, A. V. (1993). Comprometimento organizacional em uma instituição universitária. Revista de Administração, 28, 50-61. Recuperado de http://www.rausp.usp.br/busca/ artigo.asp?num_artigo $=423$.

Buckley, M., Fedor, D, Veres, J., Wiese, D., \& Carraher, S. (1998). Investigating newcomer expectations and job-related outcomes. Journal of Applied Psychology, 83, 452-461. doi: 10.1037/0021-9010.83.3.452

Burke, R., \& Macdermid, G. (1999). Are workaholics job satisfied and successful in their careers? Career Development International, 4, 277-282. doi: 10.1108/13620439910279761

Byrne, B. (2010). Structural equation modeling with AMOS. New York: Taylor \& Francis.

Carr, J., Pearson, A., Vest, M., \& Boyar, S. (2006). Prior occupational experience, anticipatory socialization, and employee retention. Journal of Management, 32, 1-17. doi: $10.1177 / 0149206305280749$.

Chay, Y., \& Aryee, S. (1999). Potential moderating influence of career growth opportunities on careerist orientation and work attitudes: Evidence of the protean career era in Singapore. Journal of Organizational Behavior, 20(5), 613-623. doi: 10.1002/ (SICI) 1099-1379(199909)20:5<613::AIDJOB979>3.0.CO;2-A

Chen, G., Ployhart, R., Thomas, H., Anderson, N., \& Bliese, P. (2011). The power of momentum: A new model of dynamic relationships between job satisfaction change and turnover intentions. Academy of Management Journal, 54, 159-181. doi: 10.5465/ AMJ.2011.59215089. 
Costa, L.V. (2014). Sucesso na carreira. Em M.M. Siqueira (Eds.). Novas medidas do comportamento organizacional (pp. 280-297). Porto Alegre: Artmed.

Damásio, B. (2012). Uso da análise fatorial exploratória em psicologia. Avaliação Psicológica, 11, 213-228. Recuperado de pepsic.bvsalud.org/scielo.php?pi$\mathrm{d}=$ S1677-04712012000200007\&script $=$ sci_arttext

De Souza, G. (2002). A study of the influence of promotions on promotion satisfaction and expectations of future promotions among managers. Human Resource Development Quaterly, 13(3), 325-340. doi: 10.1002/hrdq.1034.

Diogo, M. (2007). Os sentidos do trabalho de limpeza e conservação. Psicologia em Estudo, 12, 483-492. Recuperado de scielo.br/pdf/pe/v12n3/v12n3a05. pdf.

Eby, L., Allen, T., \& Brinley, A. (2005). A cross-level investigation of the relationship between career management practices and career-related attitudes. Group and Organization Management, 30, 565-596. doi: 10.1177/1059601104269118.

Feldman, D. C., \& Ng, T. W. (2007). Careers: Mobility, embeddedness, and success. Journal of Management, 33, 350-377. doi: 10.1177/0149206307300815.

Field, A. P. (2009). Discovering statistics using SPSS. Thousand Oaks, CA: Sage.

Gibson, D. \& Lawrence, B. (2010). Women's and men's career referents: how gender composition and comparison level shape career expectations. Organization Science, 21, 1159-1175.doi: 10.1287/ orsc.1090.0508.

Gubler, M., Arnold, J., \& Coombs, C. (2014). Reassessing the protean career concept: Empirical findings, conceptual components, and measurement. Journal of Organizational Behavior, 35(S1), S23-S40. doi: 10.1002/job.1908.

Graf, L,. \& Coutinho, M. (2010). Trajetórias de mulheres atuantes em pequenos abatedouros de animais. Cadernos de Psicologia Social do Trabalho, 13, 119-132. Recuperado de http:// pepsic.bvsalud.org/scielo.php?script=sci_arttext\&pid=S1516-37172010000100010

Greenhaus, J., \& Kossek, E. (2014). The contemporary career: A work-home perspective. Annual Review of Organizational Psychology and
Organizational Behavior, 1, 361-388. doi: 10.1146/ annurev-orgpsych-031413-091324

Hair, J. F., Black, W., Babin, B., Anderson, R., \& Tatham, R. (2010). Multivariate Data Analysis (sétima edição). Upper Saddle River, NJ: Prentice Hall.

Hall, D. (2004). The protean career: A quarter-century journey. Journal of Vocational Behavior, 65, 1-13. doi:10.1016/j.jvb.2003.10.006.

Hall, D., \& Chandler, D. (2004). Psychological success: When the career is a calling. Journal of Organizational Behavior, 25, 1-22. doi: 10.1002/job.301

Kline, R. (2011). Principles and practice of structural equation modeling. New York: Guilford Press.

Kraimer, M. L., Seibert, S. E., Wayne, S. J., Liden, R., \& Bravo, J. (2011). Antecedents and outcomes of organizational support for development: The critical role of career opportunities. Journal of Applied Psychology, 96, 485-500. doi: 10.1037/a0021452.

Lopes, A. \& Silva, J. (2009). Expectativas profissionais no discurso de terceirizados em TI. Revista de Administração de Empresas Eletrônica, 8(2), 0-0. Recuperado de http://www.scielo.br/pdf/raeel/v8n2/ v8n2a02.pdf.

O’Neill, O. A., Stanley, L. J., \& O’Reilly, C. A. (2011). Disaffected Pollyannas: The influence of positive affect on salary expectations, turnover, and long-term satisfaction. Journal of Occupational and Organizational Psychology, 84, 599-617. doi: 10.1348/096317910X500801.

Oettingen, G., \& Mayer, D. (2002). The Motivating Function of Thinking About the Future: Expectations versus Fantasies. Journal of Personality and Social Psychology, 83, 1198-1212. doi: 10.1037//0022-3514.83.5.1198.

Ourique, L. \& Teixeira, M. A. (2012). Autoeficácia e personalidade no planejamento de carreira de universitários. Psico-USF, 17(2), 311-321. doi: 10.1590/ S1413-82712012000200015.

Pasquali, L. (2010). Instrumentação Psicológica. Porto Alegre: Artmed.

Prince, J. (2003). Career opportunity and organizational attachment in a blue-collar unionized environment. Journal of Vocational Behavior, 63, 136-150. doi:10.1016/S0001-8791(02)00024-6. 
Souza, M., Pereira, P., Funck, A., \& Formiga, N. (2013). Consistência interna e estrutura fatorial da escala de expectativa de futuro em brasileiros. Boletim da Academia Paulista de Psicologia, 33(85), 330-353. Recuperado de http://www.redalyc.org/articulo. oa?id=94629531009

Stroh, L., \& Reilly, A. (1997). Rekindling Organizational Loyalty: The Role of Career Mobility. Journal of Career Development, 24, 39-54. doi: 10.1023/A:1025034504937

Super, D. E. (1980). The life span, life space approach to career development. Journal of Vocational Behavior, 16, 282-298. doi:10.1016/0001-8791(80)90056-1.

Tabachnick, B. G., \& Fidell, L. S. (2007). Using multivariate statistics. Boston: Pearson.

Tolfo, S. (2002). Carreira Profissional e seus movimentos: Revendo conceitos e formas de gestão. Revista Psicologia: Organizaçôes e Trabalho, 2(2), 39-63. Recuperado de https://periodicos.ufsc.br/index.php/ rpot/article/view/6847.

Treadway, D., Breland, J., Adams, G., Duke, A., \& Willians, L. (2010). The interactive effects of political skill and future time perspective on career and community networking behavior. Social Networks, 32, 138-147. doi:10.1016/j.socnet.2009.09.004

Vasconcellos, V., \& Neiva, E. (2014). Avaliação de futuro profissional e sua relação com bem-estar no trabalho e intenção de desligamento. Gestão e
Planejamento, 15, 410-427. Recuperado de www.spell.org.br/documentos/download/33827.

Vasconcellos, V. (2015). Antecedentes e consequentes de expectativas de carreira e de futuro organizacional (Tese de Doutorado não publicada). Instituto de Psicologia da Universidade de Brasília, Brasília, Brasil.

Veloso, E., Dutra, J., Fischer, A., Pimentel, J., Silva, R. \& Amorim, W. (2011). Gestão de carreiras e crescimento profissional. Revista Brasileira de Orientação Profissional, 12, 61-72. Recuperado de pepsic.bvsalud.org/scielo.php?pi$\mathrm{d}=$ S1679-33902011000100008\&script=sci_arttext

Veloso, E., Silva, R. D., \& Dutra, J. (2012). Diferentes gerações e percepções sobre carreiras inteligentes e crescimento profissional nas organizações. Revista Brasileira de Orientação Profissional, 13(2), 197-208. Recuperado de pepsic. bvsalud.org/scielo.php?script=sci_arttext\&pi$\mathrm{d}=$ S1679-33902012000200007.

Zacher, H., \& Frese, M. (2011). Maintaining a focus on opportunities at work: The interplay between age, job complexity, and the use of selection, optimization, and compensation strategies. Journal of Organizational Behavior, 32, 291-318. doi: 10.1002/ job.683.

Recebido em: 13/07/2015

Reformulado em: 19/08/2015

Aprovado em: 27/08/2015 
Sobre os autores:

Vinicius Carvalho de Vasconcellos é graduado em Psicologia pela UFRJ (2005), mestre em saúde pública pela Fiocruz (2008) e doutor pelo Programa de Psicologia Social, do Trabalho e das Organizações da UnB (2015). É psicólogo concursado na Petrobras, ocupando atualmente o cargo de consultor interno na área corporativa de Recursos Humanos.

E-mail: viniciuscarvalhodevasconcellos@gmail.com

Elaine Rabelo Neiva é graduada em psicologia pela UnB, mestre em Psicologia pela UnB e doutora em Psicologia pela Universidade de Brasília e Estágio Doutoral no Exterior - Doutorado Sanduiche pela Universidade Complutense de Madri, com pós-doutorado no Programa de Pós-graduação em Psicologia Social e Organizacional da Columbia - University at New York, Teacher's College. Atualmente é professora da UnB.

E-mail:elaine_neiva@uol.com.br

Contato com os autores:

A/C Vinicius Carvalho de Vasconcellos

Petrobras - Edifício Sede

Av. República do Chile, 65, Centro, Sala 702

CEP: 20031-912

Rio de Janeiro-RJ, Brasil 
